\title{
Clinical Profile of Carcinoma Breast Patients Treated with Trastuzumab: A Single Centre Study
}

\author{
Srinivasa Belagutty Jayappa ${ }^{1}$, Bhanu Prakash Lalkota1*, Veluswamy Mani², Reshma Elsa Jenny', \\ Kiran P. Krishnamurthi' ${ }^{1}$, Vinu Sarathy' ${ }^{1}$ S. Thineshwaran', Bayas Nithin ${ }^{1}$, Sumithra Martinovic ${ }^{3}$, \\ Amritanshu Ram ${ }^{2}$, Shekar Patili1, Radheshyam Naik ${ }^{1}$
}

\author{
${ }^{1}$ Department of Medical Oncology, Healthcare Global Enterprises Ltd., Bangalore, Karnataka, India \\ ${ }^{2}$ Center for Academics and Research, HCG Foundation, Bangalore, India \\ ${ }^{3}$ Department of Cytogenetics, Healthcare Global Enterprises Ltd., Bangalore, Karnataka, India \\ Email: *bhanu.lalkota@gmail.com, sripav77@gmail.com
}

How to cite this paper: Jayappa, S.B., Lalkota, B.P., Mani, V., Jenny, R.E., Krishnamurthi, K.P., Sarathy, V., Thineshwaran, S., Nithin, B., Martinovic, S., Ram, A., Patil, S. and Naik, R. (2020) Clinical Profile of Carcinoma Breast Patients Treated with Trastuzumab: A Single Centre Study. Advances in Breast Cancer Research, 9, 110-118.

https://doi.org/10.4236/abcr.2020.94009

Received: July 10, 2020

Accepted: September 12, 2020

Published: September 15, 2020

Copyright $\odot 2020$ by author(s) and Scientific Research Publishing Inc. This work is licensed under the Creative Commons Attribution International License (CC BY 4.0).

http://creativecommons.org/licenses/by/4.0/ (c) (i) Open Access

\begin{abstract}
Introduction: Breast cancer is the most common female cancer in India and accounting for almost 1 in 4 cancer cases in women worldwide. According to GLOBOCAN 2018: breast cancer incidence is increased to 162,468 in 2018 compared to 144,937 in 2012 . Biosimilar drugs allow expanding access to the therapies in the form of cost savings and leading to better overall health outcomes. Our study evaluates the efficacy and safety of Trastuzumab biosimilars and assesses overall survival in the study population. Materials \& Methods: This prospective study was conducted in Healthcare Global Enterprises Ltd., Bengaluru, India, and all female patients diagnosed with Her2 positive, metastatic $(\mathrm{mBC})$ and Locally advanced breast cancer (LABC), between March 2013 and November 2014, with at least 4 years of post-treatment follow up. Results: A total of 65 patients diagnosed with Her2 positive breast cancer and satisfied the selection criteria were included for the study. Partial Response (PR) was observed in 42 (64.6\%) patients, Stable Disease (SD) in 11 (16.9\%) patients and Progressive Disease (PD) in $12(18.5 \%)$ patients. The overall response rates were $46.1 \% \mathrm{PR}, 30 \% \mathrm{SD}, 23.8 \% \mathrm{PD}$ in metastatic population and $76 \% \mathrm{PR}, 7.2 \%$ $\mathrm{SD}, 15 \% \mathrm{PD}$ observed in locally advanced disease. The mean overall survival of the study population was $20.75 \pm 15.20$ months in metastatic and $29.2 \pm$ 17.06 months in locally advanced patients. Conclusion: This prospective study shows the effectiveness of Trastuzumab for HER2-positive in locally advanced and metastatic breast cancer. The response rates, survival and toxicity correlate with other global studies. The response and survival are as same as either generic or original Trastuzumab.
\end{abstract}




\section{Keywords}

Breast Cancer, Trastuzumab, Biosimilars

\section{Introduction}

Breast cancer is the most common female cancer in India and accounting for almost 1 in 4 cancer cases in women worldwide. According to GLOBOCAN 2018: breast cancer incidence is increased to 162,468 in 2018 compared to 144,937 in 2012 [1]. HER2/neu overexpressed in 20\% - 25\% of breast cancer patients in Indian population and worldwide [2]. HER2 overexpression is associated with an aggressive clinical phenotype that includes high-grade tumors, increased growth rates, early systemic metastasis, and decreased rates of disease-free and overall survival [3]. Addition of anti-HER2 therapy to chemotherapy, as compared with chemotherapy alone, significantly improves progression-free and overall survival among patients with HER2+ metastatic breast cancer [4]. Trastuzumab is a humanized monoclonal antibody directed to the external domain of HER2 and exerts its antitumor effects by blocking HER2 cleavage, stimulating antibody-dependent, cell-mediated cytotoxicity and inhibiting ligand-independent, HER2-mediated mitogenic signalling [5]. Initially approved by all major regulatory bodies for the treatment of HER2+ metastatic breast cancer (MBC), approved use of Trastuzumab was expanded to HER2+ early breast cancer (EBC) in 2006 [6]. Trastuzumab for 1 year (once in 21 days - 17 cycles) administered with an acceptable chemotherapy regimen is the recommended standard of care according to the guidelines [7] [8]. Clinical trials in HER2+ EBC and MBC have established that treatment with Trastuzumab/chemotherapy increases disease-free and overall survival (OS) [4] [9]. As a result, Trastuzumab has become standard of care in the treatment for Her2+ breast cancer patients. A significant side effect with this drug was cardiac dysfunction, including congestive heart failure (1\% $3 \%$ ), especially when Trastuzumab was used in combination with anthracycline-based regimens [10]. Trastuzumab related decreased Left Ventricular Ejection Fraction (LVEF) usually resolves after drug discontinuation [11]. Biosimilars are biologic medicines which are highly similar to the reference biological molecule and they demonstrated no differences in the efficacy, safety and purity [12]. Cost savings from the use of a biosimilar might allow for expanded access to the therapies, indirectly leading to better overall health outcomes. Multiple Trastuzumab biosimilars are available in the market. Our study evaluates the efficacy and safety of Trastuzumab biosimilars and assesses overall survival in the study population.

The Objectives of the study:

1) To assess the response rate to Trastuzumab therapy in metastatic and non-metastatic breast cancer patients.

2) To evaluate overall survival and toxicity in these patients. 


\section{Materials \& Methods}

This prospective study was conducted in Healthcare Global Enterprises Ltd., Bengaluru, India. The study included patients who were 20 - 70 years of age and all female patients having histologically or cytologically diagnosed as metastatic or non-metastatic breast cancer, between March 2013 and November 2014, with at least 4 years of follow up post treatment were included in the study. Estrogen Receptor (ER), Progesterone Receptor (PR), Human Epidermal Receptor (HER2), Ki-67\% expression, performed by immunohistochemistry (IHC) was obtained from patient case files. When Her2 status was 2+ by IHC, Fluorescence In-Situ Hybridization (FISH) was done to ascertain positivity. Patients who were not willing to undergo treatment with Trastuzumab and who failed to receive minimum 3 cycles of Trastuzumab or defaulted or expired during the course of treatment were excluded from the study. Hormonal Receptor status and Her2 positivity were confirmed prior to initiating chemotherapy. Also, only patients who followed standard guidelines for Trastuzumab (Herclone/Biceltis and Canmab) were included. Patients received the first dose of Trastuzumab at $8 \mathrm{mg} / \mathrm{kg}$ and subsequently, every 21 days received a dose of $6 \mathrm{mg} / \mathrm{kg}$ for 17 cycles. All the patients underwent CT/MRI/PETCT scan before and after treatment with Trastuzumab were assessed response (Partial Response-PR, Stable Disease-SD and Progressive Disease-PD). Electrocardiogram (ECG) and echocardiogram (ECHO) to assess cardiac toxicity [Left Ventricular Ejection Fraction-LVEF] before starting therapy and 3 - 6 cycles after therapy. Toxicity was considered, when LVEF drop by $>10 \%$ after treatment with Trastuzumab. All these clinical information extracted from patient case files.

\section{Statistical Analysis:}

Data was analysed using SPSS 10.0 software (Inc., Chicago, III., USA), and descriptive statistics were used to determine the patient's clinical characteristics. The chi-square test was used to compare categorical tumor features in the HER2 positive breast cancer patients. Overall Survival (OS) was the time from initial diagnosis of breast cancer to death/lost to follow-up. Kaplan Meier and log rank tests were used for the analysis of OS. For all the analyses, a $\alpha$ of $\leq 0.05$ was considered statistically significant.

\section{Results}

A total of 65 patients diagnosed with Her2 positive breast cancer and satisfied the selection criteria were included for the study. Amongst the sample, 26 (40\%) patients presented with metastatic disease and 39 (60\%) were locally advanced. The age of patients in the study was $53.23 \pm 10.79$ years. Patient's with PSO $(\mathrm{n}=$ $1,1.5 \%)$, PS1 ( $n=46,70.8 \%)$, PS2 $(n=18,27.8 \%)$. Forty-four $(68.8 \%)$ patients were post-menopausal. Hormonal positivity was observed in $n=31(47.7 \%)$ patients, hormonal negative in 34 (52.3\%). Ki-67\% expression levels were available for 65 patients and 64 (98.4\%) patients had Ki-67 > 20\%. HER2 by IHC $3+$ expressed in $45(69.2 \%)$ patients and IHC $2+$ were confirmed by FISH were 20 
(30.8\%) patients (Table 1 ).

Response was evaluated in all 65 patients who were treated with Trastuzumab. The overall response of study population in $\mathrm{mBC}$ was $46.1 \% \mathrm{PR}, 30 \% \mathrm{SD}, 23.8 \%$ $\mathrm{PD}$ and in Locally advanced it was $76 \% \mathrm{PR}, 7.2 \% \mathrm{SD}, 15 \% \mathrm{PD}$ (Table 2). In ER, PR-Her2 positive patients, the response rates in $\mathrm{mBC}$ were Partial Response (PR) 47\%, Stable Disease (SD) 35\%, Progressive Disease (PD) 17.8\% and in locally advanced, $76.4 \%$ PR, SD, $11.7 \%$ PD. Similarly, in triple positive patients, response rates in $\mathrm{mBC}$ were $44.4 \% \mathrm{PR}, 22.2 \% \mathrm{SD}, 33.3 \% \mathrm{PD}$ and in locally advanced, $77.2 \% \mathrm{PR}, 4.5 \% \mathrm{SD}, 18.1 \% \mathrm{PD}$. The response rates with Herclone/Biceltis were 55.5\% PR, 25\% SD, 20\% PD in metastatic and $87.5 \%$ PR, 8.3\% SD, 4.1\% PD in locally advanced patients. Biosimilar Canmab has shown $16.6 \%$ PR, 50\% Table 1. Baseline patient clinical characteristics of study sample.

\begin{tabular}{|c|c|c|c|}
\hline Characteristics & Overall & Metastatic & Locally Advanced \\
\hline Median Age & $53.00 \pm 10.787$ & $50.62 \pm 10.02$ & $54.97 \pm 11.06$ \\
\hline Chemo duration & 3 weekly & 3 weekly & 3 weekly \\
\hline Pre-menopausal & $20 / 65$ & $11 / 26$ & $9 / 39$ \\
\hline Post-menopausal & $45 / 65$ & $15 / 26$ & $30 / 39$ \\
\hline HER 2 by IHC $3+$ & $45 / 65$ & $17 / 26$ & $28 / 39$ \\
\hline HER2 (IHC 2+) confirmed by FISH & $20 / 65$ & $9 / 26$ & $11 / 39$ \\
\hline \multicolumn{4}{|l|}{ Hormonal status (ER, PR, HER2 neu) } \\
\hline Triple Positive & $31 / 65$ & $9 / 26$ & $22 / 39$ \\
\hline ER \& PR negative, HER2 positive & $34 / 65$ & $17 / 26$ & $17 / 39$ \\
\hline \multicolumn{4}{|l|}{ Chemotherapy regimen } \\
\hline AC $\rightarrow$ Paclitaxel + Trastuzumab & $30 / 65$ & $15 / 26$ & $25 / 39$ \\
\hline $\mathrm{TCH}$ & $35 / 65$ & $13 / 26$ & $22 / 39$ \\
\hline Ki $67>20$ & $64 / 65$ & $26 / 26$ & $38 / 39$ \\
\hline Ki $67<20$ & $1 / 65$ & $1 / 26$ & $0 / 39$ \\
\hline
\end{tabular}

Table 2. Response Rates of patient characteristics.

\begin{tabular}{|c|c|c|c|c|c|c|c|c|}
\hline \multirow{2}{*}{ Subgroups } & & \multicolumn{3}{|c|}{ Metastatic } & \multicolumn{3}{|c|}{ Locally Advanced } & \multirow{2}{*}{$\begin{array}{c}\text { p-value (Mantel } \\
\text { Cox Log Rank } \\
\text { Test) }\end{array}$} \\
\hline & & PR & SD & $\mathrm{PD}$ & PR & SD & PD & \\
\hline \multirow[t]{2}{*}{ Hormonal Subtypes } & ERPR-Her2+ & $8 / 17(47 \%)$ & $\begin{array}{c}6 / 17 \\
(35 \%)\end{array}$ & $\begin{array}{c}3 / 17 \\
(17.8 \%)\end{array}$ & $\begin{array}{c}13 / 17 \\
(76.4 \%)\end{array}$ & $\begin{array}{c}2 / 17 \\
(11.7 \%)\end{array}$ & $\begin{array}{c}2 / 17 \\
(11.7 \%)\end{array}$ & 0.190 \\
\hline & Triple Positive & $\begin{array}{c}4 / 9 \\
(44.4 \%)\end{array}$ & $\begin{array}{c}2 / 9 \\
(22.2 \%)\end{array}$ & $\begin{array}{c}3 / 9 \\
(33.3 \%)\end{array}$ & $\begin{array}{c}17 / 22 \\
(77.2 \%)\end{array}$ & $\begin{array}{c}1 / 22 \\
(4.5 \%)\end{array}$ & $\begin{array}{c}4 / 22 \\
(18.1 \%)\end{array}$ & \\
\hline \multirow[t]{2}{*}{ Trastuzumab } & Herclone/Biceltis & $\begin{array}{l}11 / 20 \\
(55 \%)\end{array}$ & $\begin{array}{c}5 / 20 \\
(25 \%)\end{array}$ & $\begin{array}{c}4 / 20 \\
(20 \%)\end{array}$ & $\begin{array}{c}21 / 24 \\
(87.5 \%)\end{array}$ & $\begin{array}{c}2 / 24 \\
(8.3 \%)\end{array}$ & $\begin{array}{c}1 / 24 \\
(4.1 \%)\end{array}$ & 0.520 \\
\hline & Canmab & $\begin{array}{c}1 / 6 \\
(16.6 \%)\end{array}$ & $\begin{array}{c}3 / 6 \\
(50 \%)\end{array}$ & $\begin{array}{c}2 / 6 \\
(33.3 \%)\end{array}$ & $\begin{array}{c}9 / 15 \\
(60 \%)\end{array}$ & $\begin{array}{c}1 / 15 \\
(6.6 \%)\end{array}$ & $\begin{array}{c}5 / 15 \\
(33.3 \%)\end{array}$ & \\
\hline Overall & response & $\begin{array}{c}12 / 26 \\
(46.1 \%)\end{array}$ & $\begin{array}{c}8 / 26 \\
(30 \%)\end{array}$ & $\begin{array}{c}6 / 26 \\
(23.8 \%)\end{array}$ & $\begin{array}{l}30 / 39 \\
(76 \%)\end{array}$ & $\begin{array}{c}3 / 39 \\
(7.2 \%)\end{array}$ & $\begin{array}{c}6 / 39 \\
(15 \%)\end{array}$ & 0.368 \\
\hline
\end{tabular}


SD, 33.3\% PD in metastatic and 60\% PR, 6.6\% SD, 33.3\% PD in locally advanced patients.

Locally advanced patients shown better overall response ( $72 \%$ vs $47.6 \%)$ and less progressive disease ( $15 \%$ vs $23.8 \%$ ) when compared to metastatic patients. The mean overall survival of the study population was $20.75 \pm 15.20$ months in metastatic and $29.2 \pm 17.06$ months in locally advanced patients. The main toxicity of Trastuzumab, cardiotoxicity (EF drop) was observed $<10 \%$ in 54 (83.1\%) and in $11(16.9 \%)$ patients (Table 3$)$. The p-value was not significant, as the study population number was small.

\section{Discussion}

The frequency of breast cancer has increased rapidly over the last decades especially in premenopausal women [13]. Breast cancer in India is known to be characterized by a higher prevalence of aggressive breast cancers with lower rates of estrogen and progesterone receptor expression [14] [15]. In a retrospective study by Ghosh et al., estrogen receptor and progesterone receptor were expressed in $56.3 \%$ and $53.1 \%$ of cases, respectively [16]. In another study by Kaul et al., estrogen and progesterone receptor positivity was noted in $34.5 \%$ and $36.4 \%$ cases, respectively [17]. Findings of the current study are in agreement with this data. It is well known that patients Trastuzumab concurrent with endocrine therapy more effective than HER2 therapy alone [18]. The addition of other anti-HER2 drugs to Trastuzumab improved median survival and overall survival [19]. In this study, patients were not treated with dual anti-HER2 therapies because of unavailability of the drugs.

The association of hormonal receptor status with the expression of $\mathrm{Ki}-67$ is a relatively active topic of research. While Madani et al. note that patients with triple negative breast cancer may have higher Ki-67 as compared to other subtypes [20]. All the patients included in the current study were HER2 positive and almost all of them (64 patients) had Ki-67 values $>20$. While this may indicate that the high expression of Ki-67 in Indian patients with breast cancer may be independent of hormonal receptor status. Bustreo et al. note that a $20 \% \mathrm{Ki}-67$ cut-off may be better than the $14 \%$ cut-off for better risk-stratification [21].

Although far from established, Ki-67 expression is known to predict pathological complete response (pCR) rates in patients with breast cancer. Patil et al. in their study noted no significant difference between clinical or pathological responders and non-responders with respect to pre-treatment or post-treatment median Ki-67 index [22]. Buzatto et al. note that addition of Trastuzumab to neoadjuvant chemotherapy significantly improves pCR rate from $4.8 \%$ to $46.8 \%$, regardless of the number of preoperative Trastuzumab cycles [23]. Furthermore,

Table 3. Toxicity of the patients who were enrolled in the study.

\begin{tabular}{cccc}
\hline Toxicity & $\leq 10 \%$ Drop & $>10 \%$ Drop & p-value (Mantel Cox Log Rank Test) \\
\hline LV Ejection Fraction & $54 / 65(83 \%)$ & $11 / 65(17 \%)$ & 0.625 \\
\hline
\end{tabular}


the study noted that hormone receptors and Ki-67 expressions were not predictive of response. About $23.8 \%$ patients in the current study achieved a pathological complete response and all of them had Ki-67 > 20\% before treatment initiation.

Results of the current study indicate that overall survival did not differ with respect to hormone-receptor status and Ki-67 expressions. Furthermore, overall survival did not differ between patients on ACTH and TCH regimen. This finding is in concordance with the findings of Reeder-Hayes et al., which notes that the 5-year survival outcomes do not differ between patients on ACTH and $\mathrm{TCH}$ regimen [24]. This study noted that patients on $\mathrm{TCH}$ regimen may be more likely to complete Trastuzumab regimen. However, in Indian settings cost presents a significant barrier to Trastuzumab in HER-2 positive breast cancer patients [15].

Although biosimilar therapies hold a promise of alleviating the cost burden on access to treatment, data on comparative efficacy and safety is relatively scarce. Results of the current study indicate that overall survival did not differ between patients on Herclone/Biceltis, and Canmab. Even, response rates in original and biosimilar Trastuzumab were not statistically significant because the numbers of patients receiving biosimilar Trastuzumab were less.

According to FDA approval on biosimilars, the response of Trastuzumab with biosimilars was not superior to the generic version Apsangikar et al. [25]., an Indian study supports the statement.

With respect to cardiac safety of Trastuzumab, it may be important to monitor ejection fraction decreases. However, as per the results of the current study overall survival of patients with $>10 \%$ drop in ejection fraction did not differ from those with $<10 \%$ drop. No cardiac related mortality is seen in this study. In the median follow-up of 3 years, the incidence of CHF is not increased.

Large cohort studies and clinical trials were established Trastuzumab efficacy; this study explored a single institution experience with small number of patients.

\section{Conclusion}

This prospective study shows the effectiveness of Trastuzumab for HER2-positive in locally advanced and metastatic breast cancer. The response rates, survival and toxicity correlate with other global studies. The response and survival are as same as either generic or original Trastuzumab.

\section{Conflicts of Interest}

The authors declare no conflicts of interest regarding the publication of this paper.

\section{References}

[1] Bray, F., Ferlay, J., Soerjomataram, I., Siegel, R.L., Torre, L.A. and Jemal, A. (2018) Global Cancer Statistics 2018: GLOBOCAN Estimates of Incidence and Mortality 
Worldwide for 36 Cancers in 185 Countries. CA: A Cancer Journal for Clinicians, 68, 394-424. https://doi.org/10.3322/caac.21492

[2] Tiwari, A., Gogia, A., Deo, S.V., Shukla, N.K., Mathur, S. and Sharma, D.N. (2017) Retrospective Study of Efficacy and Safety of Neoadjuvantdocetaxel, Carboplatin, and Trastuzumab in HER2-Positive Locally Advanced and Oligometastatic Breast Cancer: An Indian Experience. Indian Journal of Cancer, 54, 343. https://doi.org/10.4103/ijc.IJC_152_17

[3] Slamon, D., Eiermann, W., Robert, N., Pienkowski, T., Martin, M., Press, M., Mackey, J., Glaspy, J., Chan, A., Pawlicki, M. and Pinter, T. (2011) Adjuvant Trastuzumab in HER2-Positive Breast Cancer. New England Journal of Medicine, 365, 1273-1283. https://doi.org/10.1056/NEJMoa0910383

[4] Slamon, D.J., Leyland-Jones, B., Shak, S., Fuchs, H., Paton, V., Bajamonde, A., Fleming, T., Eiermann, W., Wolter, J., Pegram, M. and Baselga, J. (2001) Use of Chemotherapy plus a Monoclonal Antibody against HER2 for Metastatic Breast Cancer That Overexpresses HER2. New England Journal of Medicine, 344, 783-792. https://doi.org/10.1056/NEJM200103153441101

[5] Baselga, J., Cortés, J., Kim, S.B., Im, S.A., Hegg, R., Im, Y.H., Roman, L., Pedrini, J.L., Pienkowski, T., Knott, A. and Clark, E. (2012) Pertuzumab plus Trastuzumab plus Docetaxel for Metastatic Breast Cancer. New England Journal of Medicine, 366, 109-119. https://doi.org/10.1056/NEJMoa1113216

[6] Wilson, F.R., Coombes, M.E., Wylie, Q., Yurchenko, M., Brezden-Masley, C., Hutton, B., Skidmore, B. and Cameron, C. (2017) Herceptin ${ }^{\circledR}$ (trastuzumab) in HER2Positive Early Breast Cancer: Protocol for a Systematic Review and Cumulative Network Meta-Analysis. Systematic Reviews, 6, 196. https://doi.org/10.1186/s13643-017-0588-2

[7] Eisen, A., Fletcher, G.G., Gandhi, S., Mates, M., Freedman, O.C., Dent, S.F., et al. (2015) Optimal Systemic Therapy for Early Breast Cancer in Women: A Clinical Practice Guideline. Current Oncology, 22, S67-S81.

https://doi.org/10.3747/co.22.2320

[8] Denduluri, N., Somerfield, M.R., Eisen, A., Holloway, J.N., Hurria, A., King, T.A., et al. (2016) Selection of Optimal Adjuvant Chemotherapy Regimens for Human Epidermal Growth Factor Receptor 2 (HER2)-Negative and Adjuvant Targeted Therapy for HER2-Positive Breast Cancers: An American Society of Clinical Oncology Guideline Adaptation of the Cancer Care Ontario Clinical Practice Guideline. Journal of Clinical Oncology, 34, 2416-2427. https://doi.org/10.1200/JCO.2016.67.0182

[9] Gianni, L., Dafni, U., Gelber, R.D., Azambuja, E., Muehlbauer, S., Goldhirsch, A., et al. (2011) Treatment with Trastuzumab for 1 Year after Adjuvant Chemotherapy in Patients with HER2-Positive Early Breast Cancer: A 4-Year Follow-Up of a Randomised Controlled Trial. The Lancet Oncology, 12, 236-244. https://doi.org/10.1016/S1470-2045(11)70033-X

[10] Long, H.D., Lin, Y.E., Zhang, J.J., Zhong, W.Z. and Zheng, R.N. (2016) Risk of Congestive Heart Failure in Early Breast Cancer Patients Undergoing Adjuvant Treatment with Trastuzumab: A Meta-Analysis. The Oncologist, 21, 547-554. https://doi.org/10.1634/theoncologist.2015-0424

[11] Joensuu, H., Fraser, J., Wildiers, H., Huovinen, R., Auvinen, P., Utriainen, M., Nyandoto, P., Villman, K.K., Halonen, P., Granstam-Björneklett, H. and Lundgren, L. (2018) Effect of Adjuvant Trastuzumab for a Duration of 9 Weeks vs 1 Year with Concomitant Chemotherapy for Early Human Epidermal Growth Factor Receptor 2-Positive Breast Cancer: The SOLD Randomized Clinical Trial. JAMA Oncology, 
4, 1199-1206. https://doi.org/10.1001/jamaoncol.2018.1380

[12] US Department of Health and Human Services, US Food and Drug Administration (2015) Scientific Considerations in Demonstrating Biosimilarity to a Reference Product. US Department of Health and Human Services, Washington DC.

[13] Adusumilli, P., Konatam, M.L., Gundeti, S., Bala, S. and Maddali, L.S. (2017) Treatment Challenges and Survival Analysis of Human Epidermal Growth Factor Receptor 2-Positive Breast Cancer in Real World. Indian Journal of Medical and Paediatric Oncology: Official Journal of Indian Society of Medical \& Paediatric Oncology, 38, 22. https://doi.org/10.4103/0971-5851.203511

[14] Shet, T., Agrawal, A., Nadkarni, M., et al. (2009) Hormone Receptors over the Last 8 Years in a Cancer Referral Center in India: What Was and What Is? Indian Journal of Pathology \& Microbiology, 52, 171-174. https://doi.org/10.4103/0377-4929.48909

[15] Ghosh, J., Gupta, S., Desai, S., et al. (2011) Estrogen, Progesterone and HER2 Receptor Expression in Breast Tumors of Patients, and Their Usage of HER2-Targeted Therapy, in a Tertiary Care Centre in India. Indian Journal of Cancer, 48, 391-396. https://doi.org/10.4103/0019-509X.92245

[16] Ghosh, S., Sarkar, S., Simhareddy, S., et al. (2014) Clinico-Morphological Profile and Receptor Status in Breast Cancer Patients in a South Indian Institution. Asian Pacific Journal of Cancer Prevention, 15, 7839-7842. https://doi.org/10.7314/APJCP.2014.15.18.7839

[17] Kaul, R., Sharma, J., Minhas, S.S., et al. (2011) Hormone Receptor Status of Breast Cancer in the Himalayan Region of Northern India. Indian Journal of Surgery, 73, 9-12. https://doi.org/10.1007/s12262-010-0121-5

[18] De Laurentiis, M., et al. (2005) A Meta-Analysis on the Interaction between HER-2 Expression and Response to Endocrine Treatment in Advanced Breast Cancer. Clinical Cancer Research, 11, 4741-4748. https://doi.org/10.1158/1078-0432.CCR-04-2569

[19] Swain, S.M., et al. (2015) Pertuzumab, Trastuzumab, and Docetaxel in HER2-Positive Metastatic Breast Cancer. New England Journal of Medicine, 372, 724-734. https://doi.org/10.1056/NEJMoa1413513

[20] Madani, S.H., Payandeh, M., Sadeghi, M., et al. (2016) The Correlation between Ki-67 with Other Prognostic Factors in Breast Cancer: A Study in Iranian Patients. Indian Journal of Medical and Paediatric Oncology, 37, 95-99. https://doi.org/10.4103/0971-5851.180136

[21] Bustreo, S., Osella-Abate, S., Cassoni, P., et al. (2016) Optimal Ki67 Cut-Off for Luminal Breast Cancer Prognostic Evaluation: A Large Case Series Study with a Long-Term Follow-Up. Breast Cancer Research and Treatment, 157, 363-371. https://doi.org/10.1007/s10549-016-3817-9

[22] Patil, A.V., Singhai, R., Bhamre, R.S., et al. (2011) Ki-67 Biomarker in Breast Cancer of Indian Women. North American Journal of Medical Sciences, 3, 119-128. https://doi.org/10.4297/najms.2011.3119

[23] Buzatto, I.P., Ribeiro-Silva, A., Andrade, J.M., et al. (2017) Neoadjuvant Chemotherapy with Trastuzumab in HER2-Positive Breast Cancer: Pathologic Complete Response Rate, Predictive and Prognostic Factors. Brazilian Journal of Medical and Biological Research, 50, e5674. https://doi.org/10.1590/1414-431x20165674

[24] Reeder-Hayes, K.E., Meyer, A.M., Hinton, S.P., et al. (2017) Comparative Toxicity and Effectiveness of Trastuzumab-Based Chemotherapy Regimens in Older Women with Early-Stage Breast Cancer. Journal of Clinical Oncology, 35, 3298-3305. https://doi.org/10.1200/JCO.2016.71.4345 
[25] Apsangikar, P., Chaudhry, S., Naik, M., Deoghare, S. and Joseph, J. (2017) A Comparative Phase III Clinical Study to Evaluate Efficacy and Safety of Trasturel ${ }^{\mathrm{TM}}$ (Biosimilar Trastuzumab) and Innovator Trastuzumab in Patients with Metastatic $\mathrm{Hu}$ man Epidermal Growth Factor Receptor 2 (HER2)-Overexpressing Breast Cancer. Indian Journal of Cancer, 54, 664. https://doi.org/10.4103/ijc.IJC_449_17 